TRANSACTIONS OF THE

AMERICAN MATHEMATICAL SOCIETY

Volume 352, Number 1, Pages 71-93

$\mathrm{S}$ 0002-9947(99)02240-0

Article electronically published on May 20, 1999

\title{
EVEN VALUATIONS ON CONVEX BODIES
}

\author{
DANIEL A. KLAIN
}

\begin{abstract}
The notion of even valuation is introduced as a natural generalization of volume on compact convex subsets of Euclidean space. A recent characterization theorem for volume leads in turn to a connection between even valuations on compact convex sets and continuous functions on Grassmannians. This connection can be described in part using generating distributions for symmetric compact convex sets. We also explore some consequences of these characterization results in convex and integral geometry.
\end{abstract}

Recent interest in volume as a valuation on compact convex sets stems from Hilbert's Third Problem, which is actually an ancient problem recast in modern terms. Hilbert asked if two polytopes $P$ and $Q$ can be each cut into a finite number of pieces $P_{1}, \ldots, P_{m}$ and $Q_{1}, \ldots, Q_{m}$ with each $P_{i}$ congruent to $Q_{i}$ by a rigid Euclidean motion, provided that $P$ and $Q$ have the same volume [18]. This question was answered in the negative by Max Dehn [2, 4, 29], who found a functional on polytopes that is invariant under dissections over rigid motions, while varying in value among polytopes of equal volume. In other words, the Dehn invariant is a "simple rigid motion invariant valuation" on polytopes that is not equal to volume (under any normalization).

Dehn's solution left open the question of exactly what conditions on $P$ and $Q$ imply equidissectability over the group of rigid motions, although this problem was solved by Hadwiger in the case where only translations (and no rotations nor reflections) are permitted (see [2, 16, 17, 26, 29]).

In the course of studying this and related problems, Hadwiger discovered a characterization of Euclidean volume as a continuous rigid motion invariant simple valuation on compact convex sets, that is, a continuous rigid motion invariant valuation that vanishes on convex sets of less than full dimension. This result led in turn to a complete characterization of all continuous rigid motion invariant valuations on compact convex sets in $\mathbb{R}^{n}$ as consisting of a real $(n+1)$-dimensional vector space spanned by the intrinsic volumes (or Quermassintegrals) [16] (also $[20,21,31])$. Since many standard functionals and integral operators can be interpreted as invariant valuations (such as intrinsic volumes, mean projections, Crofton and kinematic formulas), what came to be known as Hadwiger's characterization theorem proved to be a valuable tool for generating quick and effortless proofs of many formulas and equations in integral geometry.

Unfortunately Hadwiger's original proof was long and difficult [16]. While seeking a shorter proof of Hadwiger's volume characterization, the author discovered

Received by the editors June 24, 1996 and, in revised form, September 29, 1997.

1991 Mathematics Subject Classification. Primary 52A22, 52A38, 52A39, 52B45.

Research supported in part by NSF grants \#DMS 9022140 to MSRI and \#DMS 9626688 to the author. 
that in fact fewer conditions were sufficient to characterize volume than Hadwiger originally required. In particular, complete rigid motion invariance is not necessary; it is sufficient for a continuous simple valuation to be invariant under translations and reflection through the origin $[20,21]$. The sufficiency of these invariance conditions to characterize volume motivates in turn the study of even valuations.

In the present work we pursue this line, begun in [20], by establishing a connection between continuous even valuations on compact convex sets and continuous functions on Grassmannians. We also examine some consequences to convex and integral geometry.

\section{LIST OF SYMBOLS}

\begin{tabular}{|c|c|}
\hline $\mathbb{R}^{n}$ & $n$-dimensional Euclidean space \\
\hline $\mathbb{S}^{n-1}$ & unit sphere in $\mathbb{R}^{n}$, centered at $o$ \\
\hline $\mathcal{K}^{n}$ & set of compact convex subsets of $\mathbb{R}^{n}$ \\
\hline $\mathcal{K}_{c}^{n}$ & set of symmetric sets in $\mathcal{K}^{n}$ \\
\hline$h_{K}$ & support function of $K$ \\
\hline$K+L$ & Minkowski sum of $K$ and $L$ \\
\hline$V_{n}(K)$ & $n$-volume of $K \in \mathcal{K}^{n}$ \\
\hline$V_{m}$ & $m$-th intrinsic volume \\
\hline $\operatorname{Polycon}(n)$ & set of finite unions of compact convex sets \\
\hline$d S(K, \cdot)$ & Aleksandrov-Fenchel-Jessen measure of $K$ \\
\hline$V_{n}\left(K_{1}, \ldots, K_{n}\right)$ & mixed $n$-volume of $K_{1}, \ldots, K_{n} \in \mathcal{K}^{n}$ \\
\hline $\mathcal{A}^{n}$ & $\begin{array}{l}\text { space of continuous, translation invariant, even valuations } \\
\text { on } \mathcal{K}^{n}\end{array}$ \\
\hline $\mathcal{A}_{m}^{n}$ & space of valuations in $\mathcal{A}^{n}$ that are homogeneous of degree $m$ \\
\hline$G(n, m)$ & set of $m$-dimensional subspaces of $\mathbb{R}^{n}$ \\
\hline $\operatorname{Par}(n)$ & set of finite unions of parallelotopes (w.r.t. a given basis) \\
\hline $\bar{u}$ & line segment with endpoints $u$ and $-u$ \\
\hline $\mathcal{U}^{n}$ & space of continuous translation invariant valuations on $\operatorname{Par}(n)$ \\
\hline $\mathcal{U}_{m}^{n}$ & space of valuations in $\mathcal{U}^{n}$ that are homogeneous of degree $m$ \\
\hline$\wedge^{m}\left(\mathbb{R}^{n}\right)$ & space of $m$-th exterior tensors over $\mathbb{R}^{n}$ \\
\hline$\Phi$ & $\begin{array}{l}\text { injective mapping from valuations to generating functions } \\
\text { (or vectors) }\end{array}$ \\
\hline$X^{\mu}$ & generating vector for a valuation $\mu$ \\
\hline$\wedge\left(\mathbb{R}^{n}\right)$ & exterior (Grassmann) algebra over $\mathbb{R}^{n}$ \\
\hline$C(G(n, m))$ & space of continuous real-valued functions on $G(n, m)$ \\
\hline $\mathcal{K}_{E}$ & set of compact convex subsets of $E$ \\
\hline$f_{\mu}$ & generating function of a valuation $\mu$ \\
\hline$C\left(\mathbb{S}^{n-1}\right)$ & space of continuous real-valued functions on $\mathbb{S}^{n-1}$ \\
\hline $\mathcal{D}\left(\mathbb{S}^{n-1}\right)$ & space of smooth functions on $\mathbb{S}^{n-1}$ \\
\hline $\mathcal{E}\left(\mathbb{S}^{n-1}\right)$ & space of functions on $\mathbb{S}^{n-1}$ having generating measures \\
\hline$\rho_{f}$ & generating measure of a function $f$ \\
\hline$\rho_{K}$ & generating measure of a convex set $K$ \\
\hline$T_{K}$ & generating distribution of a convex set $K\left(\right.$ on $\left.\mathbb{S}^{n-1}\right)$ \\
\hline$T_{K}^{m}$ & analogous functional to $T_{K}$ for functions on $G(n, m)$. \\
\hline $\mathcal{F}_{m}^{n}$ & domain of $T_{K}^{m}$ \\
\hline$\langle E, F\rangle$ & cosine of the angle between subspaces $E$ and $F$ \\
\hline$K \mid E$ & orthogonal projection of $K$ onto $E$ \\
\hline$\widetilde{T}_{K}^{m}$ & an alternative version of $T_{K}^{m}$ \\
\hline $\mathcal{E}_{m}^{n}$ & $\begin{array}{l}\text { space of functions on } G(n, m) \text { having generating measures; } \\
\text { domain of } \widetilde{T}_{K}^{m}\end{array}$ \\
\hline & valuation on $\mathcal{K}^{n}$ induced by $f$ under $\widetilde{T}_{K}^{m}$ \\
\hline $\operatorname{Mod}(n)$ & set of all subspaces of $\mathbb{R}^{n}$ \\
\hline$C(\operatorname{Mod}(n))$ & space of continuous real-valued functions on $\operatorname{Mod}(n)$ \\
\hline
\end{tabular}




\section{Even valuations}

Denote by $\mathcal{K}^{n}$ the collection of all compact convex subsets of $\mathbb{R}^{n}$, that is, $n$ dimensional Euclidean space. The elements of $\mathcal{K}^{n}$ are also known as convex bodies. A convex body $K$ is centered about the origin if $K$ is symmetric under reflection through the origin, that is, if $K=-K$. A convex body $K$ is symmetric or centered if there exists a translate of $K$ that is centered about the origin. Denote by $\mathcal{K}_{c}^{n}$ the collection of all symmetric convex bodies in $\mathcal{K}^{n}$.

A convex body $K \in \mathcal{K}^{n}$ is determined uniquely by its support function, $h_{K}$ : $\mathbb{S}^{n-1} \longrightarrow \mathbb{R}$, defined by $h_{K}(u)=\max _{x \in K}\{x \cdot u\}$, where $\cdot$ denotes the standard inner product on $\mathbb{R}^{n}$.

For all $K, L \in \mathcal{K}^{n}$ and all $\lambda \geq 0$, the Minkowski sum $K+\lambda L$ is defined by

$$
K+\lambda L=\{x+\lambda y: x \in K \text { and } y \in L\},
$$

and has support function $h_{K+\lambda L}=h_{K}+\lambda h_{L}$.

A sequence of convex bodies $K_{i}$ is said to converge to $K$ in the Hausdorff topology if, for all $\epsilon>0$, there exists $N>0$ such that

$$
K_{i} \subseteq K+\epsilon B \text { and } K \subseteq K_{i}+\epsilon B
$$

for all $i>N$. In this case we write $K_{i} \longrightarrow K$.

A function $\mu: \mathcal{K}^{n} \longrightarrow \mathbb{R}$ is called a valuation on $\mathcal{K}^{n}$ if $\mu(\emptyset)=0$, where $\emptyset$ is the empty set, and if

$$
\mu(K \cup L)=\mu(K)+\mu(L)-\mu(K \cap L),
$$

for all $K, L \in \mathcal{K}^{n}$ such that $K \cup L \in \mathcal{K}^{n}$ as well.

The condition that $K \cup L$ be convex may seem excessively restrictive. However, any continuous valuation $\mu$ on $\mathcal{K}^{n}$ can be extended in a unique way to the lattice Polycon $(n)$ of polyconvex subsets of $\mathbb{R}^{n}$, that is, the set of all finite unions of compact convex subsets of $\mathbb{R}^{n}$. (The lattice of polyconvex sets is also known as the convex ring.) The extension is constructed as follows: Given a valuation $\mu$ on $\mathcal{K}^{n}$, and a set $M \in \operatorname{Polycon}(n)$, express $M$ as a finite union of convex bodies,

$$
M=K_{1} \cup \cdots \cup K_{m},
$$

and then compute $\mu\left(K_{1} \cup \cdots \cup K_{m}\right)$ by iterating (1). If $\mu$ is continuous, this extension of $\mu$ turns out to be well-defined [14]. In the arguments that follow, this unique extension of $\mu$ shall allow us to consider the value of $\mu$ on all finite unions of convex bodies, whether or not such unions are actually convex.

Note that a valuation $\mu$ on $\mathcal{K}^{n}$ is said to be continuous if, for any convergent sequence $K_{i} \longrightarrow K$ in $\mathcal{K}^{n}$,

$$
\lim _{i \rightarrow \infty} \mu\left(K_{i}\right)=\mu(K) .
$$

Although a given valuation may have a well-defined extension to all of Polycon $(n)$, we do not consider convergent sequences of non-convex sets when testing a valuation $\mu$ for continuity.

A valuation $\mu$ on $\mathcal{K}^{n}$ is translation invariant if $\mu(K+x)=\mu(K)$ for all $x \in \mathbb{R}^{n}$. A valuation $\mu$ on $\mathcal{K}^{n}$ is called even if $\mu(-K)=\mu(K)$ for all $K$. If a valuation $\mu$ vanishes on all convex bodies of dimension strictly less than $n$, then $\mu$ is called a simple valuation. Finally, a valuation $\mu$ on $\mathcal{K}^{n}$ is said to be homogeneous of degree $m$, if

$$
\mu(\alpha K)=\alpha^{m} \mu(K),
$$

for all $\alpha \geq 0$ and all $K \in \mathcal{K}^{n}$. 
Denote by $\mathcal{A}^{n}$ the vector space of continuous, translation invariant, even valuations on $\mathcal{K}^{n}$, and let $\mathcal{A}_{m}^{n}$ denote the subspace of valuations in $\mathcal{A}^{n}$ that are homogeneous of degree $m$.

A well-known example of a continuous translation invariant even valuation on $\mathcal{K}^{n}$ is the volume $V_{n}$. Another example is surface area.

It turns out that the intrinsic volumes $V_{0}, V_{1}, \ldots, V_{n}$ are continuous rigid motion invariant valuations on $\mathcal{K}^{n}$ (see $[23,27],\left[31\right.$, p. 290]). The intrinsic volumes $V_{i}$ give invariant extensions of $i$-dimensional volume (on $i$-planes) to polyconvex subsets of $\mathbb{R}^{n}$, where $n \geq i$. Denote by $G(n, i)$ the set of all $i$-dimensional subspaces of $\mathbb{R}^{n}$, equipped with the invariant (Haar) measure $\xi_{i}$ normalized so that

$$
\xi_{i}(G(n, i))=\left(\begin{array}{c}
n \\
i
\end{array}\right) \frac{\omega_{n}}{\omega_{i} \omega_{n-i}},
$$

where $\omega_{i}$ is the $i$-dimensional volume of the unit ball in $\mathbb{R}^{i}$. Denote by $V_{i}$ the $i$ dimensional volume in $\mathbb{R}^{i}$. The $i$-volume $V_{i}$ is extended to the $i$-th intrinsic volume (also denoted $V_{i}$ ) on all of $\mathcal{K}^{n}$ by the mean projection formula:

$$
V_{i}(K)=\int_{G(n, i)} V_{i}(K \mid E) d \xi_{i}(E),
$$

where $K \mid E$ denotes the orthogonal projection of $K$ onto the subspace $E$. Evidently the intrinsic volume $V_{i}$ is homogeneous of degree $i$.

The valuation $V_{0}$, which takes the value 1 on all non-empty compact convex sets, extends to the Euler characteristic on the lattice of polyconvex sets (see, for example, [21, 27, 31]).

Up to a constant factor, the volume $V_{n}$ is the only continuous translation invariant even simple valuation on $\mathcal{K}^{n}$. Specifically, we have the following result [20]:

Theorem 1.1. Suppose that $\mu$ is a simple valuation on $\mathcal{K}^{n}$ that is continuous and translation invariant. Then there exists $c \in \mathbb{R}$ such that $\mu(K)+\mu(-K)=c V_{n}(K)$, for all $K \in \mathcal{K}^{n}$.

The relevance of even valuations is demonstrated by the following equivalent statement of Theorem 1.1.

Theorem 1.2 (Volume Characterization Theorem). Suppose that $\mu$ is a simple valuation on $\mathcal{K}^{n}$ that is continuous, translation invariant and even. Then there exists $c \in \mathbb{R}$ such that $\mu(K)=c V_{n}(K)$, for all $K \in \mathcal{K}^{n}$.

Theorem 1.2 fails for valuations that are not even. For example, let $\Delta$ denote the equilateral triangle in $\mathbb{R}^{2}$ with vertices at the points $(0,0),(1,0),\left(\frac{1}{2}, \frac{\sqrt{3}}{2}\right)$. The valuation $\tau$ on $\mathcal{K}^{2}$ defined by

$$
\tau(K)=V_{2}(K+\Delta)-V_{2}(K+(-\Delta))
$$

is continuous, translation invariant, and vanishes on convex bodies of dimension one. (Here $V_{2}$ denotes area in $\mathbb{R}^{2}$.) Moreover $\tau$ vanishes on all centered bodies. But $\tau(K) \neq 0$ if $K$ is not centered. This follows from the Brunn-Minkowski inequality [31, p. 309]. Alternatively, an easy direct calculation shows that $\tau(\Delta) \neq 0$. Consequently $\tau \neq c V_{2}$ for any $c \in \mathbb{R}$.

In a similar vein, a valuation $\mu$ is defined to be odd if $\mu(-K)=-\mu(K)$ for all $K \in \mathcal{K}^{n}$. In [32] Schneider proved the following theorem: 
Theorem 1.3. Suppose that $\mu$ is a simple valuation on $\mathcal{K}^{n}$ that is continuous, translation invariant and odd. Then there exists a continuous odd function $g$ : $\mathbb{S}^{n-1} \longrightarrow \mathbb{R}$ such that

for all $K \in \mathcal{K}^{n}$.

$$
\mu(K)=\int_{\mathbb{S}^{n-1}} g(u) d S(K, u)
$$

Here $\mathbb{S}^{n-1}$ denotes the unit sphere in $\mathbb{R}^{n}$, and $S(K, \cdot)$ denotes the AleksandrovFenchel-Jessen measure associated with $K$.

Since every valuation on $\mathcal{K}^{n}$ can be expressed as the sum of an even valuation and an odd valuation, Theorems 1.2 and 1.3 together characterize all continuous translation invariant simple valuations on $\mathcal{K}^{n}$.

The following theorem of McMullen [24, 26, 27] will be of use to us in the sequel.

Theorem 1.4. Let $\mu$ be a continuous translation invariant valuation on $\mathcal{K}^{n}$, homogeneous of degree $m$. If $K_{1}, \ldots, K_{p} \in \mathcal{K}^{n}$ and $\lambda_{1}, \ldots, \lambda_{p}>0$, then

$$
\mu\left(\lambda_{1} K_{1}+\cdots+\lambda_{p} K_{p}\right)=\sum_{i_{1}, \ldots, i_{m}=1}^{p} \mu\left(K_{i_{1}}, \ldots, K_{i_{m}}\right) \lambda_{i_{1}} \cdots \lambda_{i_{m}},
$$

where each symmetric coefficient $\mu\left(K_{i_{1}}, \ldots, K_{i_{m}}\right)$ depends only on the bodies $K_{i_{1}}$, $\ldots, K_{i_{m}}$. Moreover, each coefficient $\mu\left(K_{i_{1}}, \ldots, K_{i_{m}}\right)$ is a continuous translation invariant function of the bodies $K_{i_{1}}, \ldots, K_{i_{m}}$.

An important special case of Theorem 1.4 is Minkowski's expansion of the volume of a Minkowski linear combination: If $K_{1}, \ldots, K_{p} \in \mathcal{K}^{n}$ and $\lambda_{1}, \ldots, \lambda_{p}>0$, then the volume $V_{n}$ is a homogeneous polynomial in the positive variables $\lambda_{1}, \ldots, \lambda_{p}$; that is,

$$
V_{n}\left(\lambda_{1} K_{1}+\cdots+\lambda_{p} K_{p}\right)=\sum_{i_{1}, \ldots, i_{n}=1}^{p} V_{n}\left(K_{i_{1}}, \ldots, K_{i_{n}}\right) \lambda_{i_{1}} \cdots \lambda_{i_{n}} .
$$

The following theorem of McMullen implies that in order to understand and characterize translation invariant valuations, it is sufficient to consider the translation invariant homogeneous valuations [24].

Theorem 1.5. Let $\mu$ be a continuous translation invariant valuation on $\mathcal{K}^{n}$. Then there exist unique continuous translation invariant valuations $\mu_{0}, \mu_{1}, \ldots, \mu_{n}$, such that each $\mu_{i}$ is homogeneous of degree $i$, and such that

$$
\mu(K)=\sum_{i=0}^{n} \mu_{i}(K)
$$

for all $K \in \mathcal{K}^{n}$.

Note that if $\mu$ is even, then

$$
\sum_{i=0}^{n} \mu_{i}(-K) \epsilon^{i}=\mu(-\epsilon K)=\mu(\epsilon K)=\sum_{i=0}^{n} \mu_{i}(K) \epsilon^{i},
$$

for all $\epsilon>0$. It follows that if $\mu$ is even, so are the homogeneous component valuations $\mu_{i}$.

It also follows easily from Theorem 1.5 that if $\mu$ is translation invariant and homogeneous of degree $m$, then $m$ must be an integer. In particular, if $\mu(K) \neq 0$ for some set $K \in \mathcal{K}^{n}$, then necessarily $m \in\{0,1, \ldots, n\}$. Said differently, if $\mu$ is 
translation invariant and homogeneous of degree $m>n$, then $\mu(K)=0$ for all $K \in \mathcal{K}^{n}$.

While Theorems 1.4 and 1.5 are well-known for more general cases (i.e. translation invariant homogeneous valuations on compact polytopes) [26, 27], the following is true in general only for even valuations:

Proposition 1.6. Suppose that $\mu \in \mathcal{A}_{n}^{n}$. Then there exists $c \in \mathbb{R}$ such that $\mu(K)=$ $c V_{n}(K)$ for all $K \in \mathcal{K}^{n}$.

Proof. Since $\mu$ is homogeneous of degree $n$, the valuation $\mu$ restricts to the zero valuation on $(n-1)$-dimensional subspaces by Theorem 1.5. In other words, $\mu$ is simple. Proposition 1.6 then follows immediately from Theorem 1.2.

For $K, L \in \mathcal{K}^{n}$ it is well-known that the mixed volume $V_{n}(K, \ldots, K, L)$ satisfies the equation

$$
n V_{n}(\underbrace{K, \ldots, K}_{n-1}, L)=\int_{\mathbb{S}^{n-1}} h_{L}(u) d S(K, u) .
$$

See, for example, [31, p. 275].

Theorem 1.7. Suppose that $\mu \in \mathcal{A}^{n}$, and suppose that $\mu$ vanishes in dimensions less than $n-1$. Then there exists $c \in \mathbb{R}^{n}$ and sequences $\left\{L_{j}\right\},\left\{M_{j}\right\}$ of convex bodies centered at the origin such that

$$
\mu(K)=c V_{n}(K)+\lim _{j \rightarrow \infty}\left[V_{n}(\underbrace{K, \ldots, K}_{n-1}, L_{j})-V_{n}(\underbrace{K, \ldots, K}_{n-1}, M_{j})\right]
$$

for all $K \in \mathcal{K}^{n}$.

Proof. For each hyperplane $E \in G(n, n-1)$, let $\mathcal{K}_{E}$ denote the set of all compact convex subsets of $E$. Since the restriction of $\mu$ to $\mathcal{K}_{E}$ satisfies the conditions of Theorem 1.2 (in dimension $n-1$ ), there exists $c_{E} \in \mathbb{R}$ such that

$$
\mu(K)=c_{E} V_{n-1}(K)
$$

for all $K \in \mathcal{K}_{E}$.

Define $f: \mathbb{S}^{n-1} \longrightarrow \mathbb{R}$ by the equation

$$
f(u)=\frac{n c_{u^{\perp}}}{2} .
$$

Because the function $f$ is continuous and even, there exists a sequence of pairs of convex bodies $\left\{L_{j}, M_{j}\right\}$ centered about the origin such that the sequence of differences of support functions $\left\{h_{L_{j}}-h_{M_{j}}\right\}$ converges uniformly to $f$ (see [30] or [31, p. 45]).

For $j>0$ let $\mu_{j}(K)=V_{n}\left(K, \ldots, K, L_{j}\right)-V_{n}\left(K, \ldots, K, M_{j}\right)$. Then for $K \in$ $\mathcal{K}_{u^{\perp}}$, the surface measure $d S(K, \cdot)$ is concentrated at the points $u,-u \in \mathbb{S}^{n}$ with mass $V_{n-1}(K)$ at each point. Since $L_{j}, M_{j}$ are centered at the origin, we have 
$h_{L_{j}}(u)=h_{L_{j}}(-u)$, and similarly for $h_{M_{j}}$. We therefore apply (6) to compute:

$$
\begin{aligned}
\mu_{j}(K) & =V_{n}(\underbrace{K, \ldots, K}_{n-1}, L_{j})-V_{n}(\underbrace{K, \ldots, K}_{n-1}, M_{j}) \\
& =\frac{1}{n} V_{n-1}(K)\left(2 h_{L_{j}}(u)-2 h_{M_{j}}(u)\right) \\
& \rightarrow V_{n-1}(K)\left(\frac{2 f(u)}{n}\right) \text { as } j \rightarrow \infty \\
& =c_{u \perp} V_{n-1}(K) \\
& =\mu(K) .
\end{aligned}
$$

For $K \in \mathcal{K}^{n}$ define $\eta(K)=\lim _{j \rightarrow \infty} \mu_{j}(K)$. The valuation $\eta$ satisfies the equations

$$
\eta(K)=\lim _{j \rightarrow \infty}\left[V_{n}(\underbrace{K, \ldots, K}_{n-1}, L_{j})-V_{n}(\underbrace{K, \ldots, K}_{n-1}, M_{j})\right]=\int_{\mathbb{S}^{n-1}} f(u) d S(K, u) .
$$

Therefore, $\eta$ is continuous, translation invariant, even, and

$$
\eta(K)=\mu(K)
$$

for all $K$ of dimension less than $n$. It follows that the valuation $\mu-\eta$ satisfies the conditions of Theorem 1.2, so that there exists $c \in \mathbb{R}$ such that $\mu(K)-\eta(K)=$ $c V_{n}(K)$ for all $K \in \mathcal{K}^{n}$. Thus

$$
\mu(K)=c V_{n}(K)+\lim _{j \rightarrow \infty}\left[V_{n}(\underbrace{K, \ldots, K}_{n-1}, L_{j})-V_{n}(\underbrace{K, \ldots, K}_{n-1}, M_{j})\right] .
$$

The following corollary is actually a special case of a theorem of McMullen [25].

Corollary 1.8. Suppose that $\mu \in \mathcal{A}_{n-1}^{n}$. Then there exist sequences $\left\{L_{j}\right\},\left\{M_{j}\right\}$ of centered convex bodies such that

$$
\mu(K)=\lim _{j \rightarrow \infty}\left[V_{n}(\underbrace{K, \ldots, K}_{n-1}, L_{j})-V_{n}(\underbrace{K, \ldots, K}_{n-1}, M_{j})\right]
$$

for all $K \in \mathcal{K}^{n}$.

Proof. Theorem 1.7 implies that there exists $c \in \mathbb{R}^{n}$ and sequences $\left\{L_{j}\right\},\left\{M_{j}\right\}$ of centered convex bodies such that

$$
\mu(K)-\lim _{j \rightarrow \infty}\left[V_{n}(\underbrace{K, \ldots, K}_{n-1}, L_{j})-V_{n}(\underbrace{K, \ldots, K}_{n-1}, M_{j})\right]=c V_{n}(K)
$$

for all $K \in \mathcal{K}^{n}$. Since $\mu \in \mathcal{A}_{n-1}^{n}$, the left hand expression in (7) gives a valuation homogeneous of degree $n-1$. It follows that $c=0$.

Corollary 1.8 can be used to show that every centered generalized convex body has a continuous even support function (see [22]).

In [9], Goodey and Weil give a similar classification for continuous valuations that are homogeneous of degree 1 . 
Theorem 1.9. Suppose that $\mu$ is a continuous translation invariant valuation on $\mathcal{K}^{n}$. Then $\mu$ is homogeneous of degree 1 if and only if there exist sequences $\left\{L_{j}\right\}_{j=0}^{\infty}$ and $\left\{M_{j}\right\}_{j=0}^{\infty}$ in $\mathcal{K}^{n}$ such that, for all $\delta>0$,

$$
\mu(K)=\lim _{j \rightarrow \infty}\left[V_{n}(\underbrace{L_{j}, \ldots, L_{j}}_{n-1}, K)-V_{n}(\underbrace{M_{j}, \ldots, M_{j}}_{n-1}, K)\right]
$$

uniformly for all convex bodies $K \subseteq \delta B$.

If we add the condition that $\mu$ is an even valuation, it is not difficult to show that (8) holds with sequences $\left\{L_{j}\right\}$ and $\left\{M_{j}\right\}$ of symmetric bodies. For example, if $L_{j}$ and $M_{j}$ are not symmetric, then replace them with the Blaschke sums $(1 / 2)\left(L_{j} \#\left(-L_{j}\right)\right)$ and $(1 / 2)\left(M_{j} \#\left(-M_{j}\right)\right)$. If $\mu$ is even, this substitution will not change the limit in (8). (For a discussion of Blaschke sums, see [31, p. 394] or [5, p. 122].)

Theorems 1.5, 1.7, and 1.9 together give a characterization of all continuous translation invariant even valuations on $\mathcal{K}^{n}$, for $n=1,2,3$. However, the situation becomes more complicated for dimension $n \geq 4$, in part because of complications in the structure of the Grassmannian $G(n, k)$ for $n \geq 4$ and $2 \leq k \leq n-2$ (as compared to $G(n, 1) \cong G(n, n-1)$ and $\left.\mathbb{S}^{n-1}\right)$. In order to shed some light on these intermediate cases, we shall examine a connection between even valuations and functions on Grassmannians. In preparation for this, it will be useful first to understand the behavior and characterization of even valuations on a special class of compact convex sets, namely, the lattice of finite unions of parallelotopes in a fixed frame.

\section{VAluations on parallelotopes}

Choose an ordered basis of unit vectors $u_{1}, \ldots, u_{n}$ for $\mathbb{R}^{n}$, which shall remain fixed throughout this section, and let $\operatorname{Par}(n)$ denote the family of sets that are obtained by taking finite unions and intersections of parallelotopes with sides parallel to the vectors $u_{1}, \ldots, u_{n}$. Note that $\operatorname{Par}(n)$ is closed under finite unions and intersections. This follows from the fact that the intersection of two parallelotopes in $\operatorname{Par}(n)$ is a parallelotope. In other words, $\operatorname{Par}(n)$ is a distributive lattice under the operations $\cup$ and $\cap$.

A valuation $\mu$ is said to be continuous on $\operatorname{Par}(n)$, provided that

$$
\mu\left(P_{n}\right) \longrightarrow \mu(P)
$$

whenever $P_{n}, P$ are parallelotopes (and not just finite unions) and $P_{n} \longrightarrow P$ in the Hausdorff topology inherited from $\mathcal{K}^{n}$.

The object of this section is to classify all continuous translation invariant homogeneous valuations defined on $\operatorname{Par}(n)$ (see also [21]).

When studying valuations on $\operatorname{Par}(n)$ we may restrict our attention to the generating set of parallelotopes in $\mathbb{R}^{n}$ with edges parallel to the coordinate axes. Specifically, Groemer [14] (see also [21]) has shown that a valuation $\mu$ defined on parallelotopes with edges parallel to the coordinate axes admits a unique extension to a valuation on the lattice $\operatorname{Par}(n)$.

The restriction of the volume $V_{n}$ to the lattice $\operatorname{Par}(n)$ is characterized by the following theorem.

Theorem 2.1 (Volume Characterization for $\operatorname{Par}(n)$ ). Let $\mu$ be a continuous translation invariant simple valuation defined on $\operatorname{Par}(n)$. Then there exists $c \in \mathbb{R}$ such 
that $\mu(P)=c V_{n}(P)$ for all $P \in \operatorname{Par}(n)$; that is, $\mu$ is equal to the volume, up to a constant factor.

Proof. For $w \in \mathbb{R}^{n}$, denote by $\bar{w}$ the closed line segment with endpoints at $w$ and $-w$. Let $P_{0}$ denote the unit parallelotope in $\operatorname{Par}(n)$, that is, the Minkowski sum $\bar{u}_{1}+\cdots+\bar{u}_{n}$. Define a continuous invariant valuation $\nu$ on $\operatorname{Par}(n)$ by the equation

$$
\nu(P)=\mu(P)-\frac{\mu\left(P_{0}\right)}{V_{n}\left(P_{0}\right)} V_{n}(P) .
$$

Note that $\nu(P)$ vanishes in dimensions less than $n$ and that $\nu\left(P_{0}\right)=0$. We need to show that $\nu(P)=0$ for all $P \in \operatorname{Par}(n)$.

If $n=1$, then the result follows readily, since a parallelotope in $\mathbb{R}$ is merely a closed line segment. Since $\nu$ vanishes on the closed line segment $P_{0}=\bar{u}_{1}$ and on sets consisting of a single point, it follows from the inclusion-exclusion principle (1) that $\nu$ must vanish on all closed line segments of rational length. It then follows from continuity that $\nu$ vanishes on all closed line segments.

Suppose $n>1$. Recall that $\nu$ is translation invariant and vanishes on lower dimensions. Since $\nu\left(P_{0}\right)=0$, a simple cut-and-paste argument shows that $\nu\left(\frac{1}{k} P_{0}\right)=$ 0 for all integers $k>0$. Therefore, $\nu(P)=0$ for every parallelotope $P$ in $\operatorname{Par}(n)$ having rational edge lengths. This follows from the fact that such a box can be built up by stacking parallelotopes of the form $\frac{1}{k} P_{0}$ for some $k>0$. The continuity of $\nu$ now implies that $\nu(P)=0$ for every parallelotope $P$ in $\operatorname{Par}(n)$. It then follows from the inclusion-exclusion principle that $\nu(P)=0$ for all $P \in \operatorname{Par}(n)$.

For an alternative proof of Theorem 2.1, see also [15, p. 47].

A valuation $\mu$ on $\operatorname{Par}(n)$ is said to be homogeneous of degree $k>0$ if

$$
\mu(\alpha P)=\alpha^{k} \mu(P)
$$

for all $P \in \operatorname{Par}(n)$ and all $\alpha \geq 0$. Using Theorem 2.1 we are now able to index all continuous valuations on $\operatorname{Par}(n)$ that are invariant under translation and are homogeneous of degree $m$.

Denote by $\mathcal{U}^{n}$ the vector space of continuous translation invariant valuations on $\operatorname{Par}(n)$, and let $\mathcal{U}_{m}^{n}$ denote the subspace of valuations in $\mathcal{U}^{n}$ that are homogeneous of degree $m$. Recall that the exterior tensor space $\wedge^{m}\left(\mathbb{R}^{n}\right)$ is an $\left(\begin{array}{c}n \\ m\end{array}\right)$-dimensional space, spanned by the basis:

$$
\left\{u_{i_{1}} \wedge \cdots \wedge u_{i_{m}}: 1 \leq i_{1}<\cdots<i_{m} \leq n\right\} .
$$

Theorem 2.2. There exists a bijective linear map $\Phi: \mathcal{U}_{m}^{n} \longrightarrow \wedge^{m}\left(\mathbb{R}^{n}\right)$, denoted $\Phi(\mu)=X^{\mu}$, such that for all $1 \leq i_{1}<\cdots<i_{m} \leq n$ and all $P \in \operatorname{Span}\left\{u_{i_{1}}, \ldots, u_{i_{m}}\right\}$,

$$
\mu(P)=X_{\left\{i_{1}, \ldots, i_{m}\right\}}^{\mu} V_{m}(P) .
$$

Here $X_{\left\{i_{1}, \ldots, i_{m}\right\}}^{\mu}$ denotes the $u_{i_{1}} \wedge \cdots \wedge u_{i_{m}}$-coordinate of the vector $X^{\mu}$ with respect to the basis (9). We call $X^{\mu}$ the generating vector for the valuation $\mu$.

Proof. For each linear coordinate subspace $E_{i_{1}, \ldots, i_{m}}=\operatorname{Span}\left\{u_{i_{1}}, \ldots, u_{i_{m}}\right\}$ let $\operatorname{Par}\left(E_{i_{1}, \ldots, i_{m}}\right)$ denote the set of all $P \in \operatorname{Par}(n)$ with $P \subset E_{i_{1}, \ldots, i_{m}}$. Since the restriction of $\mu$ to $\operatorname{Par}\left(E_{i_{1}, \ldots, i_{m}}\right)$ satisfies the conditions of Theorem 2.1 (in dimension $m$ ), there exists $c_{i_{1}, \ldots, i_{m}} \in \mathbb{R}$ such that

$$
\mu(P)=c_{i_{1}, \ldots, i_{m}} V_{m}(P),
$$


for all $P \in \operatorname{Par}\left(E_{i_{1}, \ldots, i_{m}}\right)$. Define $X^{\mu}$ by the equation

$$
X_{\left\{i_{1}, \ldots, i_{m}\right\}}^{\mu}=c_{i_{1}, \ldots, i_{m}} .
$$

Then define $\Phi(\mu)=X^{\mu}$.

Evidently $\Phi$ is a linear map. To see that $\Phi$ is injective, suppose $\Phi(\mu)=0$. Then $\mu=0$ when restricted to subspaces of dimension $m$, so that $\mu$ satisfies the hypotheses of Theorem 2.1 in dimension $m+1$. It follows that $\mu$ is equal to a constant multiple of the $(m+1)$-dimensional volume $V_{m+1}$ when restricted to a subspace of dimension $m+1$. But $\mu$ is homogeneous of degree $m$, while $V_{m+1}$ is homogeneous of degree $m+1$. Consequently, $\mu=0$ when restricted to subspaces of dimension $m+1$, so that $\mu$ satisfies the hypotheses of Theorem 2.1 in dimension $m+2$. After $n-m$ iterations of this argument it follows that $\mu=0$ on $\operatorname{Par}(n)$.

To see that $\Phi$ is surjective, suppose $X=\left(X_{\left\{i_{1}, \ldots, i_{m}\right\}}\right) \in \wedge^{m}\left(\mathbb{R}^{n}\right)$. Define a valuation $\nu$ on $\operatorname{Par}(n)$ by

$$
\nu(P)=\sum_{1 \leq i_{1}<\cdots<i_{m} \leq n} X_{\left\{i_{1}, \ldots, i_{m}\right\}} V_{m}\left(\alpha_{i_{1}} \bar{u}_{i_{1}}+\cdots+\alpha_{i_{m}} \bar{u}_{i_{m}}\right),
$$

for all $P=\alpha_{1} \bar{u}_{1}+\cdots+\alpha_{n} \bar{u}_{n}$ (up to translation). Evidently $\nu \in \mathcal{U}_{m}^{n}$, and $\Phi(\nu)=$ $X$.

The following corollary follows immediately from the injectivity of the map $\Phi$.

Corollary 2.3. Let $\mu, \nu \in \mathcal{U}_{m}^{n}$ and suppose that $\mu(P)=\nu(P)$ for all parallelotopes $P$ of dimension $m$. Then $\mu(P)=\nu(P)$ for all $P \in \operatorname{Par}(n)$.

Remark. From the proof of Theorem 2.2 it is clear that if $\mu$ and $\nu$ are known to agree on only one $m$-dimensional parallelotope $P$ in each coordinate $m$-subspace $E$, then $\mu=\nu$ on all of $\operatorname{Par}(n)$.

While the proof of McMullen's spectral theorem 1.5 is difficult for the general case of valuations on polytopes (see [24]), an analogous result for continuous valuations on the lattice $\operatorname{Par}(n)$ is easy to prove:

Theorem 2.4. Let $\mu$ be a continuous translation invariant valuation on $\operatorname{Par}(n)$. Then there exist unique continuous translation invariant valuations $\mu_{0}, \mu_{1}, \ldots, \mu_{n}$, such that each $\mu_{m}$ is homogeneous of degree $m$, and such that

$$
\mu(P)=\sum_{m=0}^{n} \mu_{m}(P)
$$

for all $P \in \operatorname{Par}(n)$.

Proof. Let $\mu$ be a continuous translation invariant valuation on $\operatorname{Par}(n)$. Let $E_{j}$ denote the $(n-1)$-hyperplane in $\mathbb{R}^{n}$ spanned by the coordinate vectors $u_{1}, \ldots, u_{j-1}$, $u_{j+1}, \ldots, u_{n}$. The restriction of $\mu$ to $E_{j}$ is a translation invariant valuation on parallelotopes in $E_{j}$. Proceeding by induction, we may assume that, for all $P \in$ $\operatorname{Par}\left(E_{j}\right)$,

$$
\mu(P)=\sum_{m=0}^{n-1} \mu_{m}(P)
$$

where $\mu_{m}$ is a homogeneous valuation on $\operatorname{Par}\left(E_{j}\right)$ of degree $m$. Thus $\mu_{m}$ is inductively defined in each coordinate subspace $E_{j}$. 
To extend $\mu_{m}$ to all of $\operatorname{Par}(n)$, define

$$
\mu_{m}(P)=\sum_{1 \leq i_{1}<\cdots<i_{m} \leq n} \mu_{m}\left(\alpha_{i_{1}} \bar{u}_{i_{1}}+\cdots+\alpha_{i_{m}} \bar{u}_{i_{m}}\right),
$$

for all parallelotopes $P=\alpha_{1} \bar{u}_{1}+\cdots+\alpha_{n} \bar{u}_{n}$, and then extend $\mu_{m}$ to $\operatorname{Par}(n)$ by inclusion-exclusion. The valuation

$$
\mu-\sum_{m=0}^{n-1} \mu_{m}
$$

vanishes on all lower dimensional parallelotopes in $\operatorname{Par}(n)$, since any such parallelotope is contained in a hyperplane parallel to one of the hyperplanes $E_{j}$. By Theorem 2.1,

$$
\mu-\sum_{m=0}^{n-1} \mu_{m}=c_{n} V_{n}
$$

for some constant $c \in \mathbb{R}$. Setting $\mu_{n}=c_{n} V_{n}$ gives

$$
\mu=\sum_{m=0}^{n} \mu_{m}
$$

Denote by $\wedge\left(\mathbb{R}^{n}\right)$ the exterior algebra (or Grassmann algebra) on $\mathbb{R}^{n}$, that is, the direct sum $\bigoplus_{m=0}^{n} \wedge^{m}\left(\mathbb{R}^{n}\right)$. Combining Theorem 2.4 with Theorem 2.2, we obtain the following:

Theorem 2.5. There exists a bijective linear map $\Phi: \mathcal{U}^{n} \longrightarrow \wedge\left(\mathbb{R}^{n}\right)$, denoted $\Phi(\mu)=X^{\mu}$, such that

$$
\mu(P)=\sum_{m=0}^{n} \sum_{1 \leq i_{1}<\cdots<i_{m} \leq n} X_{\left\{i_{1}, \ldots, i_{m}\right\}}^{\mu} V_{m}\left(\alpha_{i_{1}} \bar{u}_{i_{1}}+\cdots+\alpha_{i_{m}} \bar{u}_{i_{m}}\right),
$$

for all $P=\alpha_{1} \bar{u}_{1}+\cdots+\alpha_{n} \bar{u}_{n}$ (up to translation).

Here $X_{\left\{i_{1}, \ldots, i_{m}\right\}}^{\mu}$ denotes the $u_{i_{1}} \wedge \cdots \wedge u_{i_{m}}$-coordinate of the $\wedge^{m}\left(\mathbb{R}^{n}\right)$-component of the vector $X^{\mu}$ with respect to the basis (9) of $\wedge^{m}\left(\mathbb{R}^{n}\right)$. The proof of Theorem 2.5 is omitted.

\section{The generating Function of an EVEn Valuation}

While some of the results of the previous sections are already known for more general valuations, the results of this section are specific to even valuations. Theorem 1.2 will lead to a fundamental connection between even valuations and continuous functions on Grassmannians.

Let $C(G(n, m))$ denote the set of all real-valued continuous functions on the Grassmannian $G(n, m)$; i.e. the set of all $m$-dimensional vector subspaces of $\mathbb{R}^{n}$. For each subspace $E \in G(n, m)$, let $\mathcal{K}_{E}$ denote the set of all compact convex subsets of $E$.

Theorem 3.1. There exists an injective linear map $\Phi: \mathcal{A}_{m}^{n} \longrightarrow C(G(n, m))$, denoted $\Phi(\mu)=f_{\mu}$, such that for all $E \in G(n, m)$ and all $K \in \mathcal{K}_{E}$,

$$
\mu(K)=f_{\mu}(E) V_{m}(K) \text {. }
$$


Note that, unlike the case of valuations on $\operatorname{Par}(n)$, we do not claim surjectivity for the map $\Phi$. The image of $\Phi$ in $C(G(n, m))$ is the topic of Section 4 .

Proof. Suppose $E \in G(n, m)$. Since the restriction of $\mu$ to $\mathcal{K}_{E}$ satisfies the conditions of Theorem 1.2 (or Prop. 1.6) in dimension $m$, there exists $c_{E} \in \mathbb{R}$ such that

$$
\mu(K)=c_{E} V_{m}(K)
$$

for all $K \in \mathcal{K}_{E}$. Define $f_{\mu}: G(n, m) \longrightarrow \mathbb{R}$ by the equation

$$
f_{\mu}(E)=c_{E},
$$

and define $\Phi(\mu)=f_{\mu}$.

Evidently $\Phi$ is a linear map. To see that $\Phi$ is injective, suppose $\Phi(\mu)=0$. Then $\mu=0$ when restricted to subspaces of dimension $m$, so that $\mu$ satisfies the hypotheses of Theorem 1.2 in dimension $m+1$. It follows that $\mu$ is equal to a constant multiple of the $(m+1)$-dimensional volume $V_{m+1}$ when restricted to a subspace of dimension $m+1$. But $\mu$ is homogeneous of degree $m$, while $V_{m+1}$ is homogeneous of degree $m+1$. Consequently, $\mu=0$ when restricted to subspaces of dimension $m+1$, so that $\mu$ satisfies the hypotheses of Theorem 1.2 in dimension $m+2$. After $n-m$ iterations of this argument it follows that $\mu=0$ on $\mathcal{K}^{n}$.

For $\mu \in \mathcal{A}_{m}^{n}$ the function $\Phi(\mu)$ is called the generating function of $\mu$. Theorem 3.1 implies that a valuation $\mu \in \mathcal{A}_{m}^{n}$ is uniquely determined by its generating function.

The following is an immediate corollary of Theorem 3.1.

Corollary 3.2. Suppose $\mu, \nu \in \mathcal{A}_{m}^{n}$, and suppose that $\mu(K)=\nu(K)$ whenever $\operatorname{dim}(K) \leq m$. Then $\mu(K)=\nu(K)$ for all $K \in \mathcal{K}^{n}$.

Proof. Since $\mu$ and $\nu$ agree when restricted to any subspace of dimension $m$, it follows that $\Phi(\mu)=\Phi(\nu)$. Since $\Phi$ is injective (by Theorem 3.1), $\mu=\nu$.

In fact, we can do even better:

Corollary 3.3. Suppose $\mu, \nu \in \mathcal{A}_{m}^{n}$, and suppose that $\mu(P)=\nu(P)$ whenever $P$ is a parallelotope of dimension $m$. Then $\mu(K)=\nu(K)$ for all $K \in \mathcal{K}^{n}$.

Proof. If $P$ has dimension $m$, then some translate of $P$ lies in a subspace $E \in$ $G(n, m)$. By Theorem 3.1,

$$
\Phi(\mu)(E) V_{m}(P)=\mu(P)=\nu(P)=\Phi(\nu)(E) V_{m}(P),
$$

so that $\Phi(\mu)(E)=\Phi(\nu)(E)$ for all $E \in G(n, m)$. In other words, $\Phi(\mu)=\Phi(\nu)$. Since $\Phi$ is injective (by Theorem 3.1), it follows that $\mu=\nu$.

Remark. From the proof of Corollary 3.3 it is clear that if $\mu$ and $\nu$ are known to agree on only one $m$-dimensional parallelotope $P$ in each $E \in G(n, m)$, then $\mu=\nu$ on all of $\mathcal{K}^{n}$.

Theorem 3.1 and Corollary 3.2 fail for valuations that are not even. For example, the valuation $\tau$ of (3) is continuous, translation invariant, homogeneous of degree 1 , and restricts to zero on all 1-dimensional subspaces. But $\tau(K) \neq 0$ if $K$ is not centered.

We continue this section with an application of Corollary 3.3 to mixed volumes. For $i \leq n$ and $K_{1}, \ldots, K_{i} \in \mathcal{K}^{n}$, denote by

$$
V_{i}\left(K_{1}\left|E, \ldots, K_{i}\right| E\right)
$$


the (i-dimensional) mixed volume of the projections of $K_{1}, \ldots, K_{i}$ onto an $i$ dimensional subspace $E$ of $\mathbb{R}^{n}$.

Theorem 3.4. Let $K_{1}, \ldots, K_{i}, K_{1}^{\prime}, \ldots, K_{i}^{\prime} \in \mathcal{K}_{c}^{n}$. The following statements are equivalent:

(i) $V_{i}\left(K_{1}\left|E, \ldots, K_{i}\right| E\right)=V_{i}\left(K_{1}^{\prime}\left|E, \ldots, K_{i}^{\prime}\right| E\right)$, for all $E \in G(n, i)$.

(ii) $V_{n}\left(K_{1}, \ldots, K_{i}, L_{1}, \ldots, L_{n-i}\right)=V_{n}\left(K_{1}^{\prime}, \ldots, K_{i}^{\prime}, L_{1}, \ldots, L_{n-i}\right)$, for all $L_{1}, \ldots L_{n-i} \in \mathcal{K}^{n}$.

Compare to Lemma 2.1 of Chakerian and Lutwak in [3].

Proof. For $E \in G(n, i)$ denote by $C_{E^{\perp}}$ the $(n-i)$-dimensional unit cube in the subspace $E^{\perp}$. Recall (or see in [31]) that

$$
V_{n}(K_{1}, \ldots, K_{i}, \underbrace{C_{E^{\perp}}, \ldots, C_{E^{\perp}}}_{n-i})=\left(\begin{array}{c}
n \\
i
\end{array}\right)^{-1} V_{i}\left(K_{1}\left|E, \ldots, K_{i}\right| E\right) .
$$

Therefore, (ii) implies (i).

To show that (i) implies (ii), define valuations $\nu_{1}$ and $\nu_{2}$ on $\mathcal{K}^{n}$ by

$$
\nu_{1}(L)=V_{n}\left(K_{1}, \ldots, K_{i}, L, \ldots, L\right)
$$

and

$$
\nu_{2}(L)=V_{n}\left(K_{1}^{\prime}, \ldots, K_{i}^{\prime}, L, \ldots, L\right)
$$

for $L \in \mathcal{K}^{n}$. Since the volume $V_{n}$ is a valuation, it follows from (5) that $\nu_{1}$ and $\nu_{2}$ are valuations. It also follows from elementary properties of $V_{n}$ and the mixed volumes that $\nu_{1}$ and $\nu_{2}$ are translation invariant, continuous, and homogeneous of degree $n-i$. Since the bodies $K_{1}, \ldots, K_{i}$ and $K_{1}^{\prime}, \ldots, K_{i}^{\prime}$ are symmetric, the valuations $\nu_{1}, \nu_{2}$ are also even.

Meanwhile, the identities (10) imply that

$$
\nu_{1}\left(C_{E^{\perp}}\right)=\left(\begin{array}{c}
n \\
i
\end{array}\right)^{-1} V_{i}\left(K_{1}\left|E, \ldots, K_{i}\right| E\right),
$$

for all $E \in G(n, i)$, and similarly for $\nu_{2}$. It then follows from (i) that $\nu_{1}\left(C_{E^{\perp}}\right)=$ $\nu_{2}\left(C_{E^{\perp}}\right)$ for all $E \in G(n, i)$. In other words, we have $\nu_{1}\left(C_{F}\right)=\nu_{2}\left(C_{F}\right)$ for all $F \in G(n, n-i)$. Corollary 3.3 now implies that $\nu_{1}=\nu_{2}$. Since the mixed volume $V_{n}$ is linear with respect to Minkowski linear combinations in each parameter, (ii) then follows for all $L_{1}, \ldots L_{n-i} \in \mathcal{K}^{n}$.

It is well-known that if $K, L \in \mathcal{K}_{c}^{n}$, then $K=L$ up to translation if and only if

$$
V_{m}(K \mid E)=V_{m}(L \mid E),
$$

for all $E \in G(n, m)$ (see for example [31]). Theorem 3.1 implies a similar assertion for general even valuations.

Theorem 3.5. Suppose $\mu \in \mathcal{A}_{m}^{n}$, and let $K, L \in \mathcal{K}^{n}$. If $V_{m}(K \mid E)=V_{m}(L \mid E)$ for all $E \in G(n, m)$, then $\mu(K \mid E)=\mu(L \mid E)$ for all $E \in G(n, m)$.

If $\Phi(\mu)$ is a non-zero function (almost everywhere) on $G(n, m)$, then the converse also holds.

Proof. This is an immediate consequence of the assertion of Theorem 3.1, namely, that

for all $K \in \mathcal{K}^{n}$ and all $E \in G(n, m)$.

$$
\mu(K \mid E)=\Phi(\mu)(E) V_{m}(K \mid E),
$$


Corollary 3.6. Suppose $\mu \in \mathcal{A}_{m}^{n}$, and suppose that $\Phi(\mu)$ is a non-zero function (almost everywhere) on $G(n, m)$. Let $K, L \in \mathcal{K}_{c}^{n}$. Then $K=L$ up to translation if and only if $\mu(K \mid E)=\mu(L \mid E)$ for all $E \in G(n, m)$.

Proof. Corollary 3.6 follows directly from Theorem 3.5 and the fact that when $K$ and $L$ are symmetric we have $K=L$ up to translation if and only if $V_{m}(K \mid E)=$ $V_{m}(L \mid E)$ for all $E \in G(n, m)$.

Remark. Corollary 3.6 does not hold for all (possibly non-symmetric) $K, L \in \mathcal{K}^{n}$.

\section{The Generating Distribution of a CONVEX SeT}

The conclusion of Theorem 3.1 motivates the following questions: Which functions in $C(G(n, i))$ are generating functions of valuations in $\mathcal{A}_{m}^{n}$ ? And how does one reconstruct a valuation, given its generating function? In order to investigate the range of the injective linear map $\Phi$ of Theorem 3.1, we recall a few facts about the generating distribution of a convex body $K$, a construction due to Weil [33, 34, 35].

Denote by $\mathcal{E}\left(\mathbb{S}^{n-1}\right)$ the space of all functions $f: \mathbb{S}^{n-1} \longrightarrow \mathbb{R}$ such that there exists a signed measure $\rho_{f}$ on $\mathbb{S}^{n-1}$ with

$$
f(u)=\int_{\mathbb{S}^{n-1}}|u \cdot v| d \rho_{f}(v) .
$$

In this case $\rho_{f}$ is called a generating measure of the function $f$.

Denote by $C\left(\mathbb{S}^{n-1}\right)$ the space of all continuous functions $f: \mathbb{S}^{n-1} \longrightarrow \mathbb{R}$, and denote by $\mathcal{D}\left(\mathbb{S}^{n-1}\right)$ the space of all smooth even functions $f: \mathbb{S}^{n-1} \longrightarrow \mathbb{R}$. Blaschke [1] (see also $[34,35])$ has shown that

$$
\mathcal{D}\left(\mathbb{S}^{n-1}\right) \subset \mathcal{E}\left(\mathbb{S}^{n-1}\right) \subset C\left(\mathbb{S}^{n-1}\right) .
$$

Recall that a compact convex set $K$ is called a zonotope if $K$ can be expressed as a finite Minkowski sum of line segments. A set $K$ is a zonoid if $K$ can be approximated by zonotopes in the Hausdorff topology on $\mathcal{K}^{n}$. Zonoids having nonempty interior are also sometimes called projection bodies. Indeed, a set $K \in \mathcal{K}^{n}$ with non-empty interior is a zonoid if and only if there exists $L \in \mathcal{K}^{n}$ and a translate $K^{\prime}$ of $K$ such that $h_{K^{\prime}}(u)=V_{n-1}\left(L \mid u^{\perp}\right)$ for all $u \in \mathbb{S}^{n-1}$. In this case we write $K^{\prime}=\Pi L$ and say that $K^{\prime}$ is the projection body of $L$.

If $K \in \mathcal{K}_{c}^{m}$ and if there exist zonoids $L, M$ such that $K+L=M$, then $K$ is called a generalized zonoid. If $K$ is a generalized zonoid centered about the origin, then its support function $h_{K}$ has a signed generating measure $\rho_{h_{K}}$ on $\mathbb{S}^{n-1}$. In this case, the generalized zonoid $K$ is a zonoid if and only if its generating measure $\rho_{h_{K}}$ is a positive measure. Generalized zonoids are of special interest because of the following fact: If $K$ is a symmetric compact convex set in $\mathbb{R}^{n}$, then there exists a sequence $\left\{K_{i}\right\}$ of generalized zonoids such that $K_{i} \longrightarrow K$.

If $K$ is a generalized zonoid centered about the origin, then $h_{K} \in \mathcal{E}\left(\mathbb{S}^{n-1}\right)$, and we denote by $\rho_{K}$ the generating measure of $h_{K}$ (instead of $\rho_{h_{K}}$ ). Note that if $K$ is a projection body, with $K=\Pi L$ for some convex body $L$, then $\rho_{K}=S(L, \cdot)$, the Aleksandrov-Fenchel-Jessen measure associated with $L$. For a thorough treatment of zonoids, generalized zonoids, and projection bodies, see [5, 10, 31, 36].

In order to generalize the notion of generating measure to all symmetric compact convex sets, we move to the level of distributions. If $K$ is centered about the origin, 
denote by $T_{K}$ the distribution defined by

$$
T_{K}(f)=\rho_{f}\left(h_{K}\right)=\int_{\mathbb{S}^{n-1}} h_{K} d \rho_{f},
$$

for all $f \in \mathcal{E}\left(\mathbb{S}^{n-1}\right)$. For $K \in \mathcal{K}_{c}^{n}$, we define $T_{K}$ similarly, after first translating the center of $K$ to the origin. Weil [33, 34] has shown that $T_{K}$ is well-defined and is continuous with respect to $K$. We call $T_{K}$ the generating distribution of the compact convex set $K$. Evidently $T_{K}=\rho_{K}$ if $K$ is a generalized zonoid, since in this case

$$
\begin{aligned}
T_{K}(f) & =\rho_{f}\left(h_{K}\right) \\
& =\int_{\mathbb{S}^{n-1}} h_{K}(u) d \rho_{f}(u) \\
& =\int_{\mathbb{S}^{n-1}} \int_{\mathbb{S}^{n-1}}|u \cdot v| d \rho_{K}(v) d \rho_{f}(u) \\
& =\int_{\mathbb{S}^{n-1}} f(v) d \rho_{K}(v) \\
& =\rho_{K}(f) .
\end{aligned}
$$

The generating distribution $T_{K}$ is a fundamental tool for the construction of a partial inverse to the mapping $\Phi: \mathcal{A}_{m}^{n} \longrightarrow C(G(n, m))$ of Theorem 3.1. For a straightforward and elegant treatment of the theory of distributions, see [28]. See also [19, pp. 33-53].

For $u_{1}, \ldots, u_{n} \in \mathbb{S}^{n-1}$ denote by $D\left(u_{1}, \ldots, u_{n}\right)$ the absolute value of determinant of the collection $\left(u_{1}, \ldots, u_{n}\right)$, that is, the volume of the parallelotope spanned by $u_{1}, \ldots, u_{n}$. Denote by $u_{1} \wedge \cdots \wedge u_{m}$ the wedge product of the vectors $u_{i}$ in the exterior tensor space $\wedge^{m}\left(\mathbb{R}^{n}\right)$. If $u_{1} \wedge \cdots \wedge u_{m} \neq 0$, then we abuse notation to denote by $u_{1} \wedge \cdots \wedge u_{m}$ the $m$-subspace spanned by the vectors $u_{i}$. Finally let $U \subseteq \mathbb{S}^{n-1} \times \cdots \times \mathbb{S}^{n-1}$ denote the (open dense) set of all linearly independent $m$-tuples of unit vectors.

For $g \in \mathcal{D}(G(n, m))$, define a function, $\widetilde{g}: \mathbb{S}^{n-1} \times \cdots \times \mathbb{S}^{n-1} \longrightarrow \mathbb{R}$ by

$$
\widetilde{g}\left(u_{1}, \ldots, u_{m}\right)=\frac{1}{m !} g\left(u_{1} \wedge \cdots \wedge u_{m}\right),
$$

for $\left(u_{1}, \ldots, u_{m}\right) \in U$ and by setting $\widetilde{g}=0$ otherwise. For $w_{1}, \ldots, w_{m} \in \mathbb{R}^{n}-$ $\{o\}$, we denote the value $\widetilde{g}\left(w_{1} /\left|w_{1}\right|, \ldots, w_{m} /\left|w_{m}\right|\right)$ by $\widetilde{g}\left(w_{1}, \ldots, w_{m}\right)$ for notational convenience.

Recall that if $K \in \mathcal{K}_{c}^{n}$, then there exists a sequence of generalized zonoids $K_{i}$, having generating measures $\rho_{K_{i}}$, such that $K_{i} \longrightarrow K$. Define

$$
T_{K}^{m}(g)=\lim _{i \rightarrow \infty} \underbrace{\int_{\mathbb{S}^{n-1}} \cdots \int_{\mathbb{S}^{n-1}}}_{m} \tilde{g}\left(u_{1}, \ldots, u_{m}\right) D\left(u_{1}, \ldots, u_{m}\right) d \rho_{K_{i}} \cdots d \rho_{K_{i}},
$$

provided this limit exists and is well-defined (independently of the choice of sequence $\left\{K_{i}\right\}$ converging to $\left.K\right)$. Denote by $\mathcal{F}_{m}^{n}$ the space of all functions $g: G(n, m) \longrightarrow \mathbb{R}$ such that the limit (13) is well-defined for all $K \in \mathcal{K}_{c}^{n}$.

Remark. Note that, unlike the functional $T_{K}$ (on $\left.\mathbb{S}^{n-1}\right)$, the linear functional $T_{K}^{m}(g)$ is not necessarily a distribution in the traditional sense of the term, since there is no reason to believe a priori that $T_{K}^{m}(g)$ is defined for all $C^{\infty}$ functions $g$. In some 
cases a problem may arise, since the product $\widetilde{g} D$ will not necessarily be smooth. Determination of the domain and the precise meaning of the functional $T_{K}^{m}(g)$ is the object of the remainder of this section.

Similarly, suppose $K_{1}, \ldots, K_{m} \in \mathcal{K}_{c}^{n}$ with generalized zonoids $\left\{K_{i j}\right\}$ such that

$$
\lim _{i \rightarrow \infty} K_{i j}=K_{j}
$$

for each $j=1, \ldots, m$. For $g \in \mathcal{F}_{m}^{n}$ define

$$
T_{K_{1}, \ldots, K_{m}}(g)=\lim _{i \rightarrow \infty} \underbrace{\int_{\mathbb{S}^{n-1}} \cdots \int_{\mathbb{S}^{n-1}}}_{m} \widetilde{g}\left(u_{1}, \ldots, u_{m}\right) D\left(u_{1}, \ldots, u_{m}\right) d \rho_{K_{i 1}} \cdots d \rho_{K_{i m}} .
$$

Recall that for all generalized zonoids $K, L$ we have $\rho_{K+L}=\rho_{K}+\rho_{L}$. An elementary combinatorial argument then implies that the limit (14) exists and that $T_{K_{1}, \ldots, K_{m}}(g)$ is well-defined if and only if $g \in \mathcal{F}_{m}^{n}$. Note that

$$
T_{K, \ldots, K}(g)=T_{K}^{m}(g)
$$

for all $g \in \mathcal{F}_{m}^{n}$.

If $K$ is a generalized zonoid with generating measure $\rho_{K}$, then

$$
T_{K}^{m}(g)=\underbrace{\int_{\mathbb{S}^{n-1}} \cdots \int_{\mathbb{S}^{n-1}}}_{m} \widetilde{g}\left(u_{1}, \ldots, u_{m}\right) D\left(u_{1}, \ldots, u_{m}\right) d \rho_{K} \cdots d \rho_{K} .
$$

In other words, the functional $T_{K}^{m}$ is a measure in this case. In particular, it follows that if $K$ is a generalized zonoid, then $T_{K}^{m}(g)$ is well-defined for all $g \in C(G(n, m))$. More generally,

$$
T_{K_{1}, \ldots, K_{m}}(g)=\underbrace{\int_{\mathbb{S}^{n-1}} \cdots \int_{\mathbb{S}^{n-1}}}_{m} \widetilde{g}\left(u_{1}, \ldots, u_{m}\right) D\left(u_{1}, \ldots, u_{m}\right) d \rho_{K_{1}} \cdots d \rho_{K_{m}},
$$

for generalized zonoids $K_{1}, \ldots, K_{m}$.

Suppose that $Z$ is a zonotope with center at the origin, that is, a Minkowski sum $Z=\bar{w}_{1}+\bar{w}_{2}+\cdots+\bar{w}_{s}$ of line segments in $\mathbb{R}^{n}$. Recall that $\bar{w}$ denotes the closed line segment with endpoints at $w$ and $-w$. Since the support function of a line segment $\bar{w}$ is given by $h_{\bar{w}}(u)=|u \cdot w|$, it follows that $\rho_{Z}$ is a finite measure with masses $\left|w_{i}\right|$ concentrated at points $\pm w_{i} /\left|w_{i}\right|$ in $\mathbb{S}^{n-1}$. Denote by $Z_{i_{1}, \ldots, i_{m}}$ the (possibly degenerate) $m$-face of $Z$ corresponding to the summands $\bar{w}_{i_{1}}, \ldots, \bar{w}_{i_{m}}$. Equation (15) implies that

$$
\begin{aligned}
T_{Z}^{m}(g) & =\underbrace{\int_{\mathbb{S}^{n-1}} \cdots \int_{\mathbb{S}^{n-1}}}_{m} \widetilde{g}\left(u_{1}, \ldots, u_{m}\right) D\left(u_{1}, \ldots, u_{m}\right) d \rho_{Z} \cdots d \rho_{Z} \\
& =\sum_{i_{1}=1}^{s} \cdots \sum_{i_{m}=1}^{s} \widetilde{g}\left(w_{i_{1}}, \ldots, w_{i_{m}}\right) D\left(w_{i_{1}} /\left|w_{i_{1}}\right|, \ldots, w_{i_{m}} /\left|w_{i_{m}}\right|\right)\left|2 w_{i_{1}}\right| \cdots\left|2 w_{i_{m}}\right|,
\end{aligned}
$$

so that

$$
T_{Z}^{m}(g)=\sum_{i_{1}=1}^{s} \cdots \sum_{i_{m}=1}^{s} \widetilde{g}\left(w_{i_{1}}, \ldots, w_{i_{m}}\right) V_{m}\left(Z_{i_{1}, \ldots, i_{m}}\right) .
$$


Recall that the $m$-volume $V_{m}$ of a Minkowski linear combination of convex bodies in $\mathbb{R}^{m}$ expands into a sum of mixed $m$-volumes according to (5). In particular,

$$
V_{m}\left(Z_{i_{1}, \ldots, i_{m}}\right)=V_{m}\left(\bar{w}_{i_{1}}+\cdots+\bar{w}_{i_{m}}\right)=m ! V_{m}\left(\bar{w}_{i_{1}}, \ldots, \bar{w}_{i_{m}}\right) .
$$

The identity (17) now becomes

$$
T_{Z}^{m}(g)=\sum_{i_{1}=1}^{s} \cdots \sum_{i_{m}=1}^{s} m ! \widetilde{g}\left(w_{i_{1}}, \ldots, w_{i_{m}}\right) V_{m}\left(\bar{w}_{i_{1}}, \ldots, \bar{w}_{i_{m}}\right) .
$$

The following proposition is a special case of a theorem of Weil [35].

Proposition 4.1. Let $E \in G(n, m)$. If $K \subseteq E$ is a zonoid and $g \in C(G(n, m))$, then

$$
T_{K}^{m}(g)=g(E) V_{m}(K) .
$$

Proof. Suppose that $Z \subseteq E$ is a zonotope, given by the Minkowski sum $Z=$ $\bar{w}_{1}+\bar{w}_{2}+\cdots+\bar{w}_{s}$, for some $w_{1}, \ldots, w_{s} \in E$. Then (17) implies that

$$
\begin{aligned}
T_{Z}^{m}(g) & =\sum_{i_{1}=1}^{s} \cdots \sum_{i_{m}=1}^{s} \widetilde{g}\left(w_{i_{1}}, \ldots, w_{i_{m}}\right) V_{m}\left(Z_{i_{1}, \ldots, i_{m}}\right) \\
& =\sum_{\substack{1 \leq i_{1}<\cdots<i_{m} \leq s\\
}} m ! \widetilde{g}\left(w_{i_{1}}, \ldots, w_{i_{m}}\right) V_{m}\left(Z_{i_{1}, \ldots, i_{m}}\right) \\
& =g(E) V_{m}(Z),
\end{aligned}
$$

since the definition (12) implies that $m ! \widetilde{g}\left(w_{i_{1}}, \ldots, w_{i_{m}}\right)=g(E)$ if the vectors $\left\{w_{i_{1}}, \ldots, w_{i_{m}}\right\}$ are independent and zero otherwise. Recall that any zonoid in $E$ can be approximated by zonotopes in $E$. The proposition then follows from the continuity of $T_{K}^{m}$ and of the intrinsic volume $V_{m}$.

Proposition 4.1 can be generalized to include all symmetric compact convex sets inside an $m$-dimensional subspace $E$. Theorem 3.1 and Proposition 4.1, along with the identities (17) and (18), suggest the following theorem.

Theorem 4.2. Suppose that $\mu \in \mathcal{A}_{m}^{n}$, and let $g$ denote the generating function of $\mu$; that is, $g=\Phi(\mu)$. Then $g \in \mathcal{F}_{m}^{n}$, with

and

$$
\mu(K)=T_{K}^{m}(g),
$$

for all $K, K_{1}, \ldots, K_{m} \in \mathcal{K}_{c}^{n}$.

Here $\mu\left(K_{1}, \ldots, K_{m}\right)$ denotes the mixed valuation generated by $\mu$, as given by Theorem 1.4.

Theorem 4.2 implies that if $g: G(n, m) \longrightarrow \mathbb{R}$ is known to be the generating function of a valuation $\mu \in \mathcal{A}_{m}^{n}$, then $g \in \mathcal{F}_{m}^{n}$; that is, $T_{K}^{m}(g)$ is well-defined for all $K \in \mathcal{K}_{c}^{n}$.

Proof. To begin, recall that for generalized zonoids $K$ the value of $T_{K}^{m}(g)$ is defined, since $T_{K}^{m}$ is a measure on the Grassmannian $G(n, m)$.

Let $u_{1}, \ldots, u_{n}$ be an independent set of unit vectors in $\mathbb{R}^{n}$, and consider the restriction of the valuation $\mu$ to the lattice $\operatorname{Par}(n)$ of (finite unions of) parallelotopes with edges parallel to $u_{1}, \ldots, u_{n}$. 
Suppose $P \in \operatorname{Par}(n)$ is the parallelotope given by the Minkowski sum

$$
P=\alpha_{1} \bar{u}_{1}+\cdots+\alpha_{n} \bar{u}_{n}
$$

of line segments. From (17) we then have

$$
T_{P}^{m}(g)=\sum_{i_{1}=1}^{n} \cdots \sum_{i_{m}=1}^{n} \widetilde{g}\left(u_{i_{1}}, \ldots, u_{i_{m}}\right) V_{m}\left(\alpha_{i_{1}} \bar{u}_{i_{1}}+\cdots+\alpha_{i_{m}} \bar{u}_{i_{m}}\right) .
$$

Since $V_{m}$ is a valuation in each $m$-dimensional coordinate subspace of $\mathbb{R}^{n}$, it follows that the functional $T_{P}^{m}(g)$ is a continuous translation invariant valuation on $\operatorname{Par}(n)$, homogeneous of degree $m$. Moreover, if $P \subseteq u_{i_{1}} \wedge \cdots \wedge u_{i_{m}}$, then

$$
T_{P}^{m}(g)=m ! \widetilde{g}\left(u_{i_{1}}, \cdots, u_{i_{m}}\right) V_{m}(P)=g\left(u_{i_{1}} \wedge \cdots \wedge u_{i_{m}}\right) V_{m}(P)=\mu(P) .
$$

It follows from Corollary 2.3 that $T_{P}^{m}(g)=\mu(P)$ for all parallelotopes in $\operatorname{Par}(n)$.

Since we could have chosen any basis $u_{1}, \ldots, u_{n}$ for $\mathbb{R}^{n}$ with which to pursue this argument, it follows that $T_{P}^{m}(g)=\mu(P)$ for all parallelotopes in $\mathbb{R}^{n}$, regardless of the basis of edges $u_{1}, \ldots, u_{n}$. Moreover, it now follows that

$$
\mu\left(P_{1}, \ldots, P_{m}\right)=\underbrace{\int_{\mathbb{S}^{n-1}} \cdots \int_{\mathbb{S}^{n-1}}}_{m} \widetilde{g}\left(u_{1}, \ldots, u_{m}\right) D\left(u_{1}, \ldots, u_{m}\right) d \rho_{P_{1}} \cdots d \rho_{P_{m}},
$$

provided that $P_{1}, \ldots, P_{m}$ are parallelotopes with respect to the same basis. In particular, if $w_{1}, \ldots, w_{m} \in \mathbb{R}^{n}$, then

$$
\begin{aligned}
& \mu\left(\bar{w}_{1}, \ldots, \bar{w}_{m}\right)=\underbrace{\int_{\mathbb{S}^{n-1}} \ldots \int_{\mathbb{S}^{n-1}}}_{m} \widetilde{g}\left(u_{1}, \ldots, u_{m}\right) D\left(u_{1}, \ldots, u_{m}\right) d \rho_{\bar{w}_{1}} \cdots d \rho_{\bar{w}_{m}} \\
& =2^{m} \widetilde{g}\left(w_{1}, \ldots, w_{m}\right) D\left(w_{1}, \ldots, w_{m}\right) \\
& =\widetilde{g}\left(w_{1}, \ldots, w_{m}\right) V_{m}\left(\bar{w}_{1}+\cdots+\bar{w}_{m}\right) \\
& =m ! \widetilde{g}\left(w_{1}, \ldots, w_{m}\right) V_{m}\left(\bar{w}_{1}, \ldots, \bar{w}_{m}\right) \text {. }
\end{aligned}
$$

For any zonotope $Z=\bar{w}_{1}+\bar{w}_{2}+\cdots+\bar{w}_{s}$, we now have

$$
\begin{aligned}
\mu(Z) & =\mu\left(\bar{w}_{1}+\bar{w}_{2}+\cdots+\bar{w}_{s}\right) \\
& =\sum_{i_{1}=1}^{s} \cdots \sum_{i_{m}=1}^{s} \mu\left(\bar{w}_{i_{1}}, \ldots, \bar{w}_{i_{m}}\right) \\
& =\sum_{i_{1}=1}^{s} \cdots \sum_{i_{m}=1}^{s} m ! \widetilde{g}\left(w_{i_{1}}, \ldots, w_{i_{m}}\right) V_{m}\left(\bar{w}_{i_{1}}, \ldots, \bar{w}_{i_{m}}\right) \\
& =T_{Z}^{m}(g)
\end{aligned}
$$

by (18). It then follows from continuity that $T_{K}^{m}(g)=\mu(K)$ whenever $K$ is a zonoid. Since the set of zonoids in $\mathcal{K}^{n}$ is closed under Minkowski addition, the identity (20) is also verified for zonoids. 
Now suppose that $K$ is a generalized zonoid. That is, suppose $K+L=M$, where $L$ and $M$ are zonoids. Then we have

$$
\begin{aligned}
\mu(K, & \left.K_{2}, \ldots, K_{m}\right)=\mu\left(M, K_{2}, \ldots, K_{m}\right)-\mu\left(L, K_{2}, \ldots, K_{m}\right) \\
= & \int_{\mathbb{S}^{n-1}} \cdots \int_{\mathbb{S}^{n-1}} \widetilde{g}\left(u_{1}, \ldots, u_{m}\right) D\left(u_{1}, \ldots, u_{m}\right) d \rho_{M} d \rho_{K_{2}} \cdots d \rho_{K_{m}} \\
& -\int_{\mathbb{S}^{n-1}} \cdots \int_{\mathbb{S}^{n-1}} \widetilde{g}\left(u_{1}, \ldots, u_{m}\right) D\left(u_{1}, \ldots, u_{m}\right) d \rho_{L} d \rho_{K_{2}} \cdots d \rho_{K_{m}} \\
= & \int_{\mathbb{S}^{n-1}} \cdots \int_{\mathbb{S}^{n-1}} \widetilde{g}\left(u_{1}, \ldots, u_{m}\right) D\left(u_{1}, \ldots, u_{m}\right) d \rho_{K} d \rho_{K_{2}} \cdots d \rho_{K_{m}} .
\end{aligned}
$$

In other words, the equation (20) holds if $K_{1}=K$ is a generalized zonoid and $K_{2}, \ldots, K_{m}$ are zonoids. Repeating this argument in each variable, we infer that (20) holds when $K_{1}, \ldots, K_{m}$ are generalized zonoids. Setting $K=K_{1}=\cdots=K_{m}$ then proves (19) for generalized zonoids.

Recall that the set of generalized zonoids in $\mathbb{R}^{n}$ is dense in the set of all symmetric compact convex sets. From the continuity of $\mu$ it now follows that

$$
T_{K}^{m}(g)=\lim _{K_{i} \rightarrow K} T_{K_{i}}^{m}(g)=\lim _{K_{i} \rightarrow K} \mu\left(K_{i}\right)=\mu(K),
$$

is well-defined, where $\left\{K_{i}\right\}$ is any sequence of generalized zonoids converging to the symmetric compact convex set $K$. In other words, $\mu(K)=T_{K}^{m}(g)$ for all $K \in \mathcal{K}_{c}^{n}$. Since $T_{K}^{m}(g)$ is defined for all $K \in \mathcal{K}_{c}^{n}$, it follows that $g \in \mathcal{F}_{m}^{n}$. The same continuity argument also verifies the mixed valuation expression (20) for all symmetric $K_{1}, \ldots, K_{m}$, thereby completing the proof of Theorem 4.2.

For subspaces $E, F \in G(n, m)$, denote by $\langle E, F\rangle$ the cosine of the "angle" between the $m$-subspaces $E$ and $F$. The value of $\langle E, F\rangle$ measures the distortion of $m$-volume upon projecting orthogonally from $E$ to $F$ (or vice versa) and is equal to the cosine of the (smaller) Euclidean angle between the straight lines in the exterior tensor space $\wedge^{m}\left(\mathbb{R}^{n}\right)$ representing the subspaces $E$ and $F$ in Plücker coordinates. If $\left\{u_{1}, \ldots, u_{m}\right\}$ is an orthonormal basis for $E$ and $\left\{w_{1}, \ldots, w_{m}\right\}$ is an orthonormal basis for $F$, then

$$
\langle E, F\rangle=\left|\operatorname{det}\left[\left(u_{i} \cdot w_{j}\right)\right]\right| .
$$

The following corollary was originally proven directly by Weil [35], but now follows immediately from Theorem 4.2.

Corollary 4.3. For all $E \in G(n, m)$ and all $K, K_{1}, \ldots, K_{m} \in \mathcal{K}_{c}^{n}$, the value $T_{K}^{m}(\langle E, F\rangle)$ is well-defined. Moreover,

$$
V_{m}(K \mid E)=T_{K}^{m}(\langle E, F\rangle)
$$

and

$$
V_{m}\left(K_{1}\left|E, \ldots, K_{m}\right| E\right)=T_{K_{1}, \ldots, K_{m}}(\langle E, F\rangle)
$$

Proof. Since $V_{m} \in \mathcal{A}_{m}^{n}$, so is the valuation given by $K \mapsto V_{m}(K \mid E)$, where $E$ is a fixed $m$-dimensional subspace. Meanwhile if $K \subseteq F$ for some $F \in G(n, m)$, then $V_{m}(K \mid E)=\langle E, F\rangle V_{m}(K)$. In other words, the map $F \mapsto\langle E, F\rangle$ is the generating function for the valuation $V_{m}(\cdot \mid E)$ given by Theorem 3.1. It follows from Theorem 4.2 that $T_{K}^{m}(\langle E, F\rangle)$ is well-defined. Equations $(21)$ and $(22)$ then also follow from Theorem 4.2 . 


\section{An Alternative APPROACH}

In view of Theorem 4.2, one is tempted to conjecture that $\mathcal{A}_{m}^{n}=\left\{T_{(\cdot)}^{m}(g): g \in\right.$ $\left.\mathcal{F}_{m}^{n}\right\}$, in other words, that the map $\Phi$ is a bijection between the set $\mathcal{A}_{m}^{n}$ of valuations and the space $\mathcal{F}_{m}^{n}$ of functions. While this may be true, there remains to show that the functional $K \mapsto T_{K}^{m}(g)$ is indeed a valuation on all of $\mathcal{K}^{n}$, for every $g \in \mathcal{F}_{m}^{n}$.

Another question also arises: what exactly is $\mathcal{F}_{m}^{n}$ ? What conditions must we place on a function $g \in C(G(n, m))$ so that $T_{K}^{m}(g)$ is well-defined for all $K \in \mathcal{K}_{c}^{n}$ ? It is not clear that $T_{K}^{m}(g)$ is defined for arbitrary symmetric $K$ even when $g$ is smooth.

In order to investigate further the space $\mathcal{F}_{m}^{n}$, we continue with yet another interpretation of the generating distribution of a compact convex set $K$.

Denote by $\mathcal{E}_{m}^{n}$ the set of all functions $f: G(n, m) \longrightarrow \mathbb{R}$ such that

$$
f(E)=\int_{G(n, m)}\langle E, F\rangle d \rho_{f}(F)
$$

for some (signed) measure $\rho_{f}$ on $G(n, m)$. In this case we call $\rho_{f}$ a generating measure for $f$.

Recall that if $K$ is a compact convex subset of $\mathbb{R}^{n}$ and $E$ is an $m$-dimensional subspace of $\mathbb{R}^{n}$, then the orthogonal projection $K \mid E$ is a compact convex subset of $E$. Moreover, the support function $h_{K \mid E}$ of $K \mid E$ is given by the restriction of $h_{K}$ to $E$. For fixed $K \in \mathcal{K}^{n}$ the function $V_{m}(K \mid E)$ is called the $m$-th projection function of $K$.

For all $K \in \mathcal{K}^{n}$ define the $m$-th generating distribution $\widetilde{T}_{K}^{m}$ on $\mathcal{E}_{m}^{n}$ by

$$
\widetilde{T}_{K}^{m}(f)=\int_{G(n, m)} V_{m}(K \mid E) d \rho_{f} .
$$

We shall see that, although the generating measure $\rho_{f}$ is not necessarily unique (there may be other measures that generate the function $f$ ), the value of $\widetilde{T}_{K}^{m}(f)$ is well-defined independently of the choice of admissible $\rho_{f}$.

Note also that unlike $T_{K}$ and $T_{K}^{m}$, the functional $\widetilde{T}_{K}^{m}$ is defined not only for symmetric $K$, but for all compact convex $K$. Evidently $\widetilde{T}_{K}^{m}$ is a continuous translation invariant even valuation in the parameter $K$, homogeneous of degree $m$. This follows immediately from the fact that $V_{m}(K \mid E)$ is such a valuation in the parameter $K$ for each fixed $E$. For $f \in \mathcal{E}_{m}^{n}$, denote $\mu_{f}(K)=\widetilde{T}_{K}^{m}(f)$.

Theorem 5.1. For $f \in \mathcal{E}_{m}^{n}$, the valuation $\mu_{f}$ is well-defined. Moreover, the generating function of the valuation $\mu_{f}$ is the function $f$ itself. In other words, $\Phi\left(\mu_{f}\right)=$ $f$.

Proof. Suppose $f \in \mathcal{E}_{m}^{n}$, and let $\rho_{f}$ be a generating measure for $f$. If $K \subseteq F$ for some $F \in G(n, m)$, then $V_{m}(K \mid E)=\langle E, F\rangle V_{m}(K)$. Therefore

$$
\begin{aligned}
\mu_{f}(K) & =\widetilde{T}_{K}^{m}(f) \\
& =\int_{G(n, m)} V_{m}(K \mid E) d \rho_{f} \\
& =\int_{G(n, m)}\langle E, F\rangle V_{m}(K) d \rho_{f} \\
& =V_{m}(K) f(E) .
\end{aligned}
$$


It then follows from Theorem 3.1 that $f$ is the generating function of $\mu_{f}$.

Next, suppose $\bar{\rho}_{f}$ is another generating measure for $f$. Let $\bar{\mu}_{f}$ denote the valuation obtained by replacing $\rho_{f}$ with $\bar{\rho}_{f}$. The same argument implies that $f$ is the generating function of $\bar{\mu}_{f}$. In other words, the two valuations $\mu_{f}$ and $\bar{\mu}_{f}$ have the same generating function. Theorem 3.1 then implies that $\bar{\mu}_{f}=\mu_{f}$. In other words, the valuation $\mu_{f}$ is well-defined, independently of the choice of generating measure $\rho$ for a given function $f \in \mathcal{E}_{m}^{n}$.

As one might hope, the two functionals $T_{K}^{m}$ and $\widetilde{T}_{K}^{m}$ coincide on $\mathcal{E}_{m}^{n}$.

Theorem 5.2. For $g \in \mathcal{E}_{m}^{n}$ and $K \in \mathcal{K}_{c}^{n}$,

$$
\widetilde{T}_{K}^{m}(g)=T_{K}^{m}(g)
$$

In particular, $\mathcal{E}_{m}^{n} \subseteq \mathcal{F}_{m}^{n}$.

Proof. Suppose $g \in \mathcal{E}_{m}^{n}$, and define $\mu_{g}(K)=\widetilde{T}_{K}^{m}(g)$ for all $K \in \mathcal{K}^{n}$. By Theorem 5.1, we have $\Phi\left(\mu_{g}\right)=g$. It then follows from Theorem 4.2 that $g \in \mathcal{F}_{m}^{n}$, and that

$$
T_{K}^{m}(g)=\mu_{g}(K)=\widetilde{T}_{K}^{m}(g),
$$

for all $K \in \mathcal{K}_{c}^{n}$.

Remark. Note that $f \in \mathcal{E}_{m}^{n}$ if and only if the function $f$ can be approximated by linear combinations of absolute inner product functions $F \mapsto\langle E, F\rangle$. Consequently an even valuation $\mu$ has generating function $f_{\mu} \in \mathcal{E}_{m}^{n}$ if and only if $\mu$ can be approximated by linear combinations of projection functions $K \mapsto V_{m}(K \mid E)$. There remains the interesting question of whether $\mathcal{E}_{m}^{n}=\mathcal{F}_{m}^{n}$; that is, are all continuous even valuations (homogeneous of degree $m$ ) approximable by linear combinations of projection functions? Or if not, do there exist easily verified conditions on $\mu$ that determine whether $f_{\mu} \in \mathcal{E}_{m}^{n}$ ?

Recent developments in the study of projection functions $[7,8]$ and generalized (Grassmannian) cosine transforms $[6,11,12,13]$ may lead to interesting new examples of valuation generating functions, which may in turn shed light on the question of how better to characterize the families $\mathcal{E}_{m}^{n}$ and $\mathcal{F}_{m}^{n}$.

\section{EVEn VAluAtions REVisited}

McMullen's spectral theorem for translation invariant valuations, Theorem 1.5, leads to more general forms for Theorems 3.1, 4.2-5.2.

Denote by $\operatorname{Mod}(n)$ the set of all subspaces of $\mathbb{R}^{n}$. The set $\operatorname{Mod}(n)$ consists of the disjoint union

$$
\operatorname{Mod}(n)=\{0\} \uplus G(n, 1) \uplus G(n, 2) \uplus \cdots \uplus\left\{\mathbb{R}^{n}\right\} .
$$

For $f: \operatorname{Mod}(n) \longrightarrow \mathbb{R}$, denote by $\left.f\right|_{G(n, m)}$ the restriction of $f$ to $G(n, m)$.

Theorem 6.1. There exists an injective map $\Phi: \mathcal{A}^{n} \longrightarrow C(\operatorname{Mod}(n))$, denoted $\Phi(\mu)=f_{\mu}$, such that

$$
\mu(K)=\sum_{m=0}^{n} T_{K}^{m}\left(\left.f_{\mu}\right|_{G(n, m)}\right)
$$

for all $K \in \mathcal{K}_{c}^{n}$. 
Proof. Suppose $\mu \in \mathcal{A}^{n}$. By Theorem 1.5, there exist unique valuations $\mu_{m} \in \mathcal{A}_{m}^{n}$, for $m=0, \ldots, n$, such that

$$
\mu(K)=\sum_{m=0}^{n} \mu_{m}(K)
$$

for all $K \in \mathcal{K}^{n}$. In other words,

$$
\mathcal{A}^{n}=\mathcal{A}_{0}^{n} \oplus \mathcal{A}_{1}^{n} \oplus \cdots \oplus \mathcal{A}_{n}^{n}
$$

Since Theorem 3.1 gives an injective linear map $\Phi: \mathcal{A}_{m}^{n} \longrightarrow C(G(n, m))$ for each $m=0,1, \ldots n$, define $\Phi: \mathcal{A}^{n} \longrightarrow C(\operatorname{Mod}(n))$ to be the direct sum of these component maps. Evidently this extension of $\Phi$ remains injective.

If $K \in \mathcal{K}_{c}^{n}$ with generating distribution $T_{K}$, then

$$
\mu(K)=\sum_{m=0}^{n} \mu_{m}(K)=\sum_{m=0}^{n} T_{K}^{m}\left(\left.f_{\mu}\right|_{G(n, m)}\right),
$$

by Theorem 4.2 applied to each $\mu_{m}$.

Once again the valuation $\mu$ is determined uniquely by its values on parallelotopes.

Theorem 6.2. Let $\mu, \nu \in \mathcal{A}^{n}$, and suppose that $\mu(P)=\nu(P)$ for all parallelotopes $P$ in $\mathbb{R}^{n}$. Then $\mu(K)=\nu(K)$ for all $K \in \mathcal{K}^{n}$.

Proof. If $P$ is a parallelotope, then

$$
\sum_{m=0}^{n} \mu_{m}(P) \epsilon^{m}=\sum_{m=0}^{n} \mu_{m}(\epsilon P)=\mu(\epsilon P)=\nu(\epsilon P)=\sum_{m=0}^{n} \nu_{m}(P) \epsilon^{m},
$$

for all $\epsilon>0$. It follows that $\mu_{m}(P)=\nu_{m}(P)$ for all parallelotopes. By Corollary 3.3, each $\mu_{m}=\nu_{m}$, so that $\mu=\nu$.

Remark. It is sufficient for the valuations $\mu, \nu$ to agree on the dilates of a single (full-dimensional) parallelotope in each orthogonal frame of $\mathbb{R}^{n}$ to imply that $\mu=\nu$. The proof is similar to that of Theorem 6.2.

\section{ACKNOWLEDGEMENTS}

The author is grateful to Eric Grinberg, Rolf Schneider, and the anonymous referee for their valuable comments and suggestions.

\section{REFERENCES}

[1] W. Blaschke. Kreis und Kugel, Leipzig, 1916.

[2] V. Boltianskii. Hilbert's Third Problem. (New York: John Wiley \& Sons, 1978). MR 58:18074

[3] G. D. Chakerian and E. Lutwak. Bodies with similar projections, Trans. Amer. Math. Soc., 349 (1997), 1811-1820. MR 98a:52011

[4] M. Dehn. Über den Rauminhalt, Math. Ann., 55 (1901), 465-478.

[5] R. Gardner. Geometric Tomography (New York: Cambridge University Press, 1995). MR 96j:52006

[6] P. Goodey and R. Howard. Processes of flats induced by higher dimensional processes, Adv. Math., 80 (1990), 92-109. MR 91d:60025

[7] P. Goodey, R. Schneider, and W. Weil. Projection functions on higher rank Grassmannians, Geometric Aspects of Functional Analysis, J. Lindenstrauss and V. D. Milman, Eds., Oper. Theory Adv. Appl., $7 \mathbf{7}$ (1995), 75-90. MR 96h:52007

[8] P. Goodey, R. Schneider, and W. Weil. Projection functions of convex bodies. Intuitive Geometry (Budapest, 1995), Bolyai Soc. Math. Stud., 6 (1997), 23-53. MR 98k:52020

[9] P. Goodey and W. Weil. Distributions and valuations, Proc. London Math. Soc., Series 3, 49 (1984), 504-516. MR 86f:52008 
[10] P. Goodey and W. Weil. Zonoids and generalisations, Handbook of Convex Geometry, Peter M. Gruber and Jörg M. Wills, Eds. (Amsterdam: North-Holland, 1993), 1297-1326. MR 95g:52015

[11] E. Grinberg. Radon transforms on higher rank Grassmannians, J. Differential Geom., 24 (1986), 53-68. MR 87m:22023

[12] E. Grinberg. Cosine and Radon transforms on Grassmannians. Preprint.

[13] E. Grinberg and G. Zhang. Convolutions, transforms and convex bodies, Proc. London Math. Soc. (3), 78 (1999), 77-115.

[14] H. Groemer. On the extension of additive functionals on classes of convex sets, Pacific J. Math., 75 (1978), 397-410. MR 58:24003

[15] H. Hadwiger. Altes und Neues über konvexe Körper. (Basel: Birkhäuser Verlag, 1955). MR 17:401e

[16] H. Hadwiger. Vorlesungen über Inhalt, Oberfläche, und Isoperimetrie. (Berlin: Springer Verlag, 1957). MR 21:1561

[17] H. Hadwiger and P. Glur. Zerlegungsgleichheit ebener Polygone, Elem. Math., 6 (1951), 97-106. MR 13:576k

[18] D. Hilbert. Mathematical Problems. Lecture delivered before the International Congress of Mathematicians in Paris, 1900. Translated by M. W. Newson, Bull. Amer. Math. Soc., 8 (1902), 437-479.

[19] L. Hörmander. The Analysis of Linear Partial Differential Operators I, 2nd edition. (New York: Springer-Verlag, 1990). MR 91m:35001b

[20] D. Klain. A short proof of Hadwiger's characterization theorem, Mathematika, 42 (1995), 329-339. MR 97e:52008

[21] D. Klain and G.-C. Rota. Introduction to Geometric Probability. (New York: Cambridge University Press, 1997. CMP 98:09

[22] E. Lutwak. Extended affine surface area, Adv. Math., 85 (1991), 39-68. MR 92d:52012

[23] P. McMullen. Non-linear angle-sum relations for polyhedral cones and polytopes, Math. Proc. Camb. Phil. Soc., 78 (1975), 247-261. MR 52:15238

[24] P. McMullen. Valuations and Euler-type relations on certain classes of convex polytopes, Proc. London Math. Soc., 35 (1977), 113-135. MR 56:6548

[25] P. McMullen. Continuous translation invariant valuations on the space of compact convex sets, Arch. Math., 34 (1980), 377-384. MR 81m:52013

[26] P. McMullen. Valuations and dissections, Handbook of Convex Geometry, Peter M. Gruber and Jörg M. Wills, Eds. (Amsterdam: North-Holland, 1993), 933-988. MR 95f:52018

[27] P. McMullen and R. Schneider. Valuations on convex bodies, Convexity and Its Applications, Peter M. Gruber and Jörg M. Wills, Eds. (Boston: Birkhäuser Verlag, 1983), 170-247. MR 85e:52001

[28] I. Richards and H. Youn. Theory of Distributions: a non-technical introduction. (New York: Cambridge University Press, 1990). MR 91f:46003

[29] C.-H. Sah. Hilbert's Third Problem: Scissors Congruence. (San Francisco: Fearon Pitman Publishers Inc., 1979). MR 81g:51011

[30] R. Schneider. Equivariant endomorphisms of the space of convex bodies, Trans. Amer. Math. Soc., 194 (1974), 53-78. MR 50:5633

[31] R. Schneider. Convex Bodies: The Brunn-Minkowski Theory. (New York: Cambridge University Press, 1993). MR 94d:52007

[32] R. Schneider. Simple valuations on convex bodies, Mathematika, 43 (1996), 32-39. MR 97f:52016

[33] W. Weil. Kontinuierliche Linearkombination von Strecken, Math. Z., 148 (1976), 71-84. MR 53:3887

[34] W. Weil. Centrally symmetric convex bodies and distributions, Israel J. Math., 24 (1976), 352-367. MR 54:8450

[35] W. Weil. Centrally symmetric convex bodies and distributions II, Israel J. Math., 32 (1979), 173-182. MR 80g:52003

[36] W. Weil. Stereology: A survey for geometers, Convexity and Its Applications, Peter M. Gruber and Jörg M. Wills, Eds. (Boston: Birkhäuser Verlag, 1983). MR 85e:52007 0160

School of Mathematics, Georgia Institute of Technology, Atlanta, Georgia 30332 -

E-mail address: klain@math.gatech.edu 